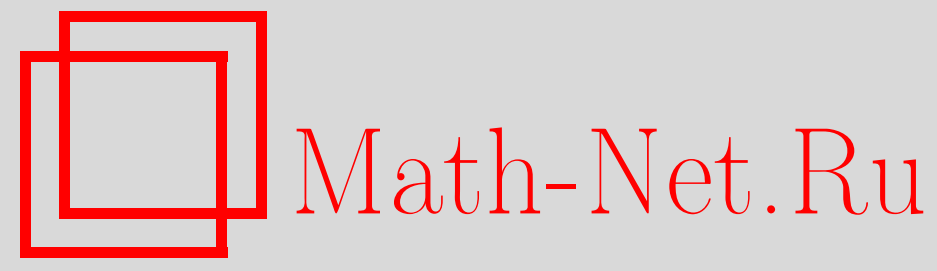

Ю. А. Неретин, Некоторые непрерывные аналоги разложения по многочленам Якоби и векторнозначные ортогональные базисы, Функи. анализ и его прил., 2005, том 39, выпуск 2, 31-46

DOI: https://doi.org/10.4213/faa38

Использование Общероссийского математического портала MathNet.Ru подразумевает, что вы прочитали и согласны с пользовательским соглашением

http://www . mathnet.ru/rus/agreement

Параметры загрузки:

IP : 54.237 .59 .107

26 апреля 2023 г., 11:55:46

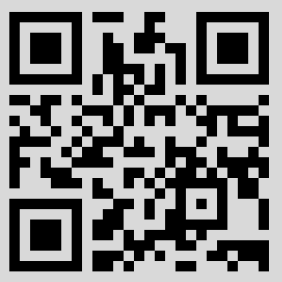


Функииональньй анализ и его приложения

2005, т. 39, вып. 2, с. 31-46

УДК 517.587

\title{
Некоторые непрерывные аналоги разложения по многочленам Якоби \\ и векторнозначные ортогональные базисы
}

\author{
(c) 2005. Ю. А. НеРЕтин

\section{§1. Теоретико-представленческая мотивировка и формулировка результатов}

1.1. Непрерывные аналоги разложения по многочленам Якоби. Настоящая работа является спецфункциональным аналогом работы Молчанова [17] о тензорных произведениях унитарных представлений группы $\mathrm{SL}_{2}(\mathbb{R})$.

В классическом анализе хорошо известно разложение по многочленам Якоби и его непрерывный аналог - индексное гипергеометрическое преобразование Г. Вейля [33] (оно также называется преобразованием Олевского [23], преобразованием Якоби, обобщенным преобразованием Фурье; см. обзор Коорнвиндера [13], а также [8,12,20]). Эти классические конструкции имеют важный теоретико-представленческий смысл (см. [32]). Сферические функции проективных пространств

$$
\mathrm{O}(n+1) / \mathrm{O}(n) \times \mathrm{O}(1), \quad \mathrm{U}(n+1) / \mathrm{U}(n) \times \mathrm{U}(1), \quad \mathrm{Sp}(n+1) / \mathrm{Sp}(n) \times \mathrm{Sp}(1)
$$

над $\mathbb{R}, \mathbb{C}$ и телом кватернионов $\mathbb{H}$ являются многочленами Якоби $P_{m}^{\alpha, \beta}$ при некоторых специальных значениях параметров $\alpha, \beta$. Задача о разложении пространства $L^{2}$ на этих однородных пространствах в прямую сумму неприводимых представлений сводится к теореме о разложении функции в ряд по многочленам Якоби.

В случае гиперболических пространств

$$
\mathrm{O}(n, 1) / \mathrm{O}(n) \times \mathrm{O}(1), \quad \mathrm{U}(n, 1) / \mathrm{U}(n) \times \mathrm{U}(1), \quad \mathrm{Sp}(n, 1) / \mathrm{Sp}(n) \times \mathrm{Sp}(1)
$$

аналогичную роль (см. $[13,23])$ играет индексное гипергеометрическое преобразование

$$
g(s)=\frac{1}{\Gamma(b+c)} \int_{0}^{\infty} f(x){ }_{2} F_{1}(b+i s, b-i s ; b+c ;-x) x^{b+c-1}(1+x)^{b-c} d x .
$$

Задача о разложении пространства $L^{2}$ на этих пространствах сводится к формуле обращения

$$
f(x)=\frac{1}{\pi \Gamma(b+c)} \int_{0}^{\infty} g(s){ }_{2} F_{1}(b+i s, b-i s ; b+c ;-x)\left|\frac{\Gamma(b+i s) \Gamma(c+i s)}{\Gamma(2 i s)}\right|^{2} d s
$$

для этого интегрального преобразования.

Однако этих двух классических конструкций - многочленов Якоби и индексного преобразования - недостаточно для изучения анализа на псевдоримановых симметрических пространствах ранга 1 (этот класс пространств включает 
в себя, в частности, другие вещественные формы пространств (1.1)-(1.2)). Это заставляет предположить, что существует какой-то иной аналог (или аналоги) индексного преобразования (1.3). Мы (одно) такое преобразование строим ${ }^{12}{ }^{2}$.

1.2. Задача о векторнозначных базисах. Хорошо известна иерархия Аски-Вильсона гипергеометрических ортогональных многочленов, см. [11] и [1, Ch. 6]. Почти все (и скорее всего, все) эти многочлены встречаются в теории представлений группы $\mathrm{SL}_{2}(\mathbb{R})$ (см., например, $\left.[31,28,29]\right)$.

Рассмотрим тензорное произведение унитарного представления группы $\mathrm{SL}_{2}(\mathbb{R})$ со старшим весом на представление с младшим весом. В каждом сомножителе есть канонический ортогональный базис, состоящий из $\mathrm{SO}(2)$-собственных функций; поэтому есть и канонический базис в тензорном произведении. Соответственно возникает канонический ортогональный базис в спектральном разложении тензорного произведения. Этот базис состоит из непрерывных двойственных многочленов Хана (см. [34]). Напомним (см. [1, 6.10] и [11]), что это многочлены $p_{n}\left(s^{2}\right)$, ортогональные по мере

$$
\left|\frac{\Gamma(a+i s) \Gamma(b+i s) \Gamma(c+i s)}{\Gamma(2 i s)}\right|^{2} d s
$$

на прямой $s \in \mathbb{R}$; они задаются формулой

$$
p_{n}\left(s^{2}\right)=(a+b)_{n}(a+c)_{n 3} F_{2}\left[\begin{array}{c}
-n, a+i s, a-i s \\
a+b, a+c
\end{array} ; 1\right] .
$$

Но та же задача имеет смысл для любой пары унитарных представлений группы $\mathrm{SL}_{2}(\mathbb{R})$ или ее универсальной накрывающей. Эти тензорные произведения, вообще говоря, имеют двукратные спектры (см. [26,17]). Таким образом, мы должны получить ортогональные базисы, состоящие из $\mathbb{C}^{2}$-значных функций.

1.3. Обозначения. Мы используем стандартные обозначения для символа Похгаммера $(a)_{n}=a(a+1) \cdots(a+n-1)$ и для гипергеометрических функций

$$
{ }_{2} F_{1}[a, b ; c ; z]:=\sum_{n=0}^{\infty} \frac{(a)_{n}(b)_{n}}{(c)_{n} n !} z^{n}, \quad{ }_{3} F_{2}\left[\begin{array}{c}
a, b, c \\
d, e
\end{array} ; z\right]:=\sum_{n=0}^{\infty} \frac{(a)_{n}(b)_{n}(c)_{n}}{(d)_{n}(e)_{n} n !} z^{n} .
$$

1.4. Двойное индексное гипергеометрическое преобразование. Фиксируем $0 \leqslant \alpha \leqslant 1 / 2, \beta \in \mathbb{R}$. Предположим, что $\alpha+i \beta \neq 0$.

1) Молчанов [17-19] использует другой метод. Ниже мы вводим дифференциальный оператор (2.1). Он возникает при ограничении оператора Лапласа (см. наш §4) в тензорном произведении унитарных представлений универсальной накрывающей группы $\mathrm{SL}_{2}(\mathbb{R}) \sim$ группы $\mathrm{SL}_{2}(\mathbb{R})$ на подпространства, собственные относительно группы вращений $\mathrm{SO}(2)$. Молчанов в [17] (он рассматривает только саму группу $\mathrm{SL}_{2}(\mathbb{R})$ ) ограничивает оператор Лапласа на функции, инвариантные относительно группы диагональных матриц. В итоге у него получается дифференциальный оператор Лежандра на некотором контуре, содержащем особые точки уравнения Лежандра.

2) Было бы интересно понять, достаточно ли классического индексного преобразования (в варианте [5, XIII] или [8]) и построенного ниже в разд. 1.4 двойного индексного преобразования для получения другого доказательства формулы Планшереля [18] на псевдоримановых симметрических пространствах $G / H$ ранга 1 . Точнее, пусть $K$ - максимальная компактная подгруппа в $G$, и пусть $V$ - неприводимый $K$-модуль. Рассмотрим действие оператора Лапласа на $\operatorname{Hom}_{K}\left(V, L^{2}(G / H)\right)$. Верно ли, что мы во всех случаях получим гипергеометрический дифференциальный оператор (2.3) на отрезке $[0,1]$ (классический случай) или на контуре $\operatorname{Re} z=1 / 2$ (наш случай)? 
Рассмотрим пространство $\mathbb{C}^{2}$-значных функций на полупрямой $s>0$. Элемент такого пространства можно рассматривать как пару скалярнозначных функций $\left(\varphi_{1}(s), \varphi_{2}(s)\right)$. Введем в этом пространстве скалярное произведение

$$
\begin{aligned}
\left\langle\left(\varphi_{1}, \varphi_{2}\right),\left(\psi_{1}, \psi_{2}\right)\right\rangle=\frac{1}{2 \pi} \int_{0}^{\infty} & {\left[r_{11}(s) \varphi_{1}(s) \overline{\psi_{1}(s)}+r_{12}(s) \varphi_{1}(s) \overline{\psi_{2}(s)}\right.} \\
& \left.+r_{21}(s) \varphi_{2}(s) \overline{\psi_{1}(s)}+r_{22}(s) \varphi_{2}(s) \overline{\psi_{2}(s)}\right] \frac{d s}{|\Gamma(2 i s)|^{2}},
\end{aligned}
$$

где $r_{i j}(s)$ задаются формулой

$$
\begin{aligned}
R(s) & =\left(\begin{array}{ll}
r_{11}(s) & r_{12}(s) \\
r_{21}(s) & r_{22}(s)
\end{array}\right) \\
& :=\left(\begin{array}{cc}
\Gamma\left(\frac{1}{2}-\alpha-i s\right) \Gamma\left(\frac{1}{2}-\alpha+i s\right) & \Gamma\left(\frac{1}{2}-i \beta-i s\right) \Gamma\left(\frac{1}{2}-i \beta+i s\right) \\
\Gamma\left(\frac{1}{2}+i \beta-i s\right) \Gamma\left(\frac{1}{2}+i \beta+i s\right) & \Gamma\left(\frac{1}{2}+\alpha-i s\right) \Gamma\left(\frac{1}{2}+\alpha+i s\right)
\end{array}\right) .
\end{aligned}
$$

Удобно записывать это скалярное произведение в векторном виде:

$$
\left\langle\left(\varphi_{1}, \varphi_{2}\right),\left(\psi_{1}, \psi_{2}\right)\right\rangle=\frac{1}{2 \pi} \int_{0}^{\infty}\left(\varphi_{1}(s) \quad \varphi_{2}(s)\right) R(s)\left(\overline{\frac{\psi_{1}(s)}{\psi_{2}(s)}}\right) \frac{d s}{|\Gamma(2 i s)|^{2}} .
$$

Обозначим полученное гильбертово пространство $\mathbb{C}^{2}$-значных функций через через $H_{\alpha, \beta}$.

Пусть $x \in \mathbb{R}, s \geqslant 0$. Рассмотрим две функции $Q_{1}(\alpha, \beta ; x, s), Q_{2}(\alpha, \beta ; x, s)$, заданные формулами ${ }^{1)}$

$$
\begin{aligned}
Q_{1}(\alpha, \beta ; x, s)= & \frac{1}{\Gamma(\alpha+i \beta)}\left(\frac{1}{2}+i x\right)^{-(\alpha+i \beta) / 2}\left(\frac{1}{2}-i x\right)^{(\alpha+i \beta) / 2-i s-1 / 2} \\
& \times{ }_{2} F_{1}\left[\begin{array}{c}
1 / 2-\alpha+i s, 1 / 2-i \beta+i s \\
1-\alpha-i \beta
\end{array} ; \frac{i x+1 / 2}{i x-1 / 2}\right], \\
Q_{2}(\alpha, \beta ; x, s)= & Q_{1}(-\alpha,-\beta ; x, s) .
\end{aligned}
$$

Для функции $f \in L^{2}(\mathbb{R})$ мы определим пару функций $\varphi_{1}(s), \varphi_{2}(x)$ по формуле

$$
\varphi_{j}(s):=\int_{-\infty}^{\infty} f(x) \overline{Q_{j}(\alpha, \beta ; x, s)} d x, \quad j=1,2 .
$$

TEOPEMA 1.1. Пусть $0 \leqslant \alpha<1 / 2, \alpha+i \beta \neq 0$.

(а) Oператор $f \mapsto\left(\varphi_{1}, \varphi_{2}\right)$ является унитарным биективным оператором из $L^{2}(\mathbb{R})$ в $H_{\alpha, \beta}$.

(b) Формула обращения имеет вид

$$
f(x):=\frac{1}{2 \pi} \int_{0}^{\infty}\left(Q_{1}(\alpha, \beta ; x, s) \quad Q_{2}(\alpha, \beta ; x, s)\right) R(s)\left(\begin{array}{l}
\varphi_{1}(s) \\
\varphi_{2}(s)
\end{array}\right) \frac{d s}{|\Gamma(2 i s)|^{2}} .
$$

ЗАмечАниЕ. Пусть $A(s)$ - функция на $\mathbb{R}$, принимающая значения в $2 \times 2$-матрицах. Любая калибровка вида

$$
\left(Q_{1}^{\circ}(s), Q_{2}^{\circ}(s)\right):=\left(Q_{1}(s), Q_{2}(s)\right) A(s)^{-1}, \quad R^{\circ}(s):=A(s)^{*} R(s) A(s)
$$

1) Это собственные функции введенного ниже дифференциального оператора (2.1), почти лежащие в $L^{2}$.

2 Функциональный анализ и его приложения, т. 39, вып. 2 
дает другую форму нашего интегрального преобразования. Было бы интересно найти функции $A(s)$, для которых матрица $R^{\circ}(s)$ и вектор $\left(Q_{1}^{\circ}(s), Q_{2}^{\circ}(s)\right)$ остаются относительно простыми.

1.5. Обобщение на случай $\boldsymbol{\alpha}>\mathbf{1} / \mathbf{2}$. Пусть теперь $\alpha>1 / 2, \beta \in \mathbb{R}$ и $\alpha+i \beta \neq 0,1,2, \ldots$. Обозначим через $n$ целую часть числа $\alpha-1 / 2$. Рассмотрим конечномерное линейное пространство $W_{\alpha, \beta}$, состоящее из векторов $\left(c_{0}, c_{1}, \ldots, c_{n}\right)$. Скалярное произведение в $W_{\alpha, \beta}$ мы зададим формулой

$$
\left\langle c, c^{\prime}\right\rangle=\frac{1}{2 \pi} \sum_{k=0}^{n} \frac{2 \alpha-2 k-1}{\Gamma(2 \alpha-k) k !} c_{k} \bar{c}_{k}^{\prime} .
$$

Далее введем функции ${ }^{1)}$

$$
\begin{aligned}
& R(\alpha, \beta ; x ; k) \\
& :=\frac{1}{\Gamma(\alpha+i \beta)}\left(\frac{1}{2}+i x\right)^{-(\alpha+i \beta) / 2}\left(\frac{1}{2}-i x\right)^{-(\alpha-i \beta) / 2}{ }_{2} F_{1}\left[\begin{array}{c}
-k, k-2 \alpha+1 \\
1-\alpha-i \beta
\end{array} ; \frac{1}{2}+i x\right] .
\end{aligned}
$$

Теперь мы рассмотрим линейный оператор

$$
J_{\alpha, \beta}: L^{2}(\mathbb{R}) \rightarrow H_{\alpha, \beta} \oplus W_{\alpha, \beta},
$$

заданный формулой

$$
f \mapsto\left(\varphi_{1}, \varphi_{2}, \theta\right),
$$

где $\varphi_{1}, \varphi_{2}$, как и выше, задаются формулой (1.7), а координаты вектора $\theta \in$ $W_{\alpha, \beta}$ имеют вид

$$
\theta_{k}=\int_{-\infty}^{\infty} f(x) \overline{R(\alpha, \beta ; x ; k)} d x .
$$

Tеорема 1.2. Оператор $J_{\alpha, \beta}$ является унитарным биективным оператором.

1.6. Многочлены Романовского. Многочлены Романовского [27] — это многочлены на $\mathbb{R}$, ортогональные по весу

$$
\left(\frac{1}{2}+i x\right)^{-(\alpha+i \beta)}\left(\frac{1}{2}-i x\right)^{-(\alpha-i \beta)} d x .
$$

Этот вес убывает со скоростью $x^{-2 \alpha}$, и поэтому можно ортогонализовать лишь конечное число степеней $1, x, x^{2}, \ldots$ Многочлены Романовского задаются формулой

$$
{ }_{2} F_{1}\left[-k, k-2 \alpha+1 ; 1-\alpha-i \beta ; \frac{1}{2}+i x\right],
$$

т. е., с точностью до элементарного множителя, формулой (1.9).

Напомним, что многочлены Якоби задаются формулой

$$
P_{n}^{\gamma, \delta}(x)=\mathrm{const} \cdot{ }_{2} F_{1}[-n, n+\gamma+\delta, \delta+1 ;(1+x) / 2]
$$

Мы видим, что многочлены Романовского являются аналитическими продолжениями многочленов Якоби по верхним индексам.

Таких конечных систем известно довольно много, см. [2, 14-16,3,21], некоторые приложения рассматриваются в $[24,3,4]$. Такого рода многочлены являются собственными функциями некоторых дифференциальных или разностных

1)Это собственные функции введенного ниже дифференциального оператора (2.1), лежащие в $L^{2}$. 
операторов второго порядка. Соответственно мы получаем хороший набор спектральных задач (что, в свою очередь, должно дать набор «новых» интегральных преобразований $)^{1)}$. Недавно одна из самых сложных задач такого рода (связанная с ${ }_{4} F_{3}$-многочленами из [21]) была решена Груневельтом [9].

1.7. Векторнозначные базисы. Фиксируем параметры $0<\alpha<1 / 2, \beta, p$, $q \in \mathbb{R}$. Мы рассматриваем гильбертово пространство $Y(\alpha, \beta ; q)$, состоящее из $\mathbb{C}^{2}$-значных функций на полуоси $s \geqslant 0$, со скалярным произведением

$$
\begin{aligned}
& \left\langle\left(\varphi_{1}, \varphi_{2}\right),\left(\psi_{1}, \psi_{2}\right)\right\rangle \\
& =\frac{1}{2} \int_{0}^{\infty}\left(\varphi_{1}(s) \quad \varphi_{2}(s)\right) R(s)\left(\overline{\frac{\psi_{1}(s)}{\psi_{2}(s)}}\right)\left|\frac{\Gamma(1+i q+i s) \Gamma(1-i q+i s)}{\Gamma(2 i s)}\right|^{2} d s
\end{aligned}
$$

где матрица $R$ - та же, что и выше (1.5).

Далее, определим функции $\Xi_{n}^{(1)}(\alpha, \beta ; p, q ; s), \Xi_{n}^{(2)}(\alpha, \beta ; p, q ; s)$ от переменной $s$ формулами

$$
\begin{aligned}
& \Xi_{n}^{(1)}(\alpha, \beta ; p, q ; s)=\frac{\cos ((p-\alpha-i q+i \beta) \pi / 2) \Gamma(1-\alpha+i \beta)}{\Gamma(\alpha-i \beta) \Gamma(1+i q+i \beta) \Gamma(1+i q-\alpha)} \\
& \quad \times{ }_{3} F_{2}\left[\begin{array}{c}
(1-\alpha+i \beta-p+i q) / 2-n, 1 / 2+i q+i s, 1 / 2+i q-i s \\
1+i q+i \beta, 1+i q-\alpha
\end{array}\right],
\end{aligned}
$$

$\Xi_{n}^{(2)}(\alpha, \beta ; p, q ; s)=\Xi_{n}^{(1)}(-\alpha,-\beta ; p, q ; x)$.

ЗАмЕчАниЕ. Гипергеометрический ряд ${ }_{3} F_{2}\left(a_{1}, a_{2}, a_{2} ; b_{1}, b_{2} ; 1\right)$ сходится абсолютно при $\sum a_{i}<\sum b_{j}$ и продолжается как мероморфная (однозначная) функция на произвольные значения параметров $a_{1}, a_{2}, a_{2}, b_{1}, b_{2}$. Мы понимаем (1.12) как значение этой мероморфной функции. Ниже, в (3.4), функция $\Xi_{n}^{(1)}$ представлена в виде гипергеометрического ряда, который сходится при всех интересующих нас значениях параметров.

ТЕоремА 1.3. Система функций $\left(\Xi_{n}^{(1)}, \Xi_{n}^{(2)}\right)$, где $n$ пробегает $\mathbb{Z}$, является ортонормированным базисом гильбертова пространства $Y(\alpha, \beta ; q)$.

ЗАмечАниЕ. Отметим, что выражение (1.12) имеет структуру

$$
\text { const } \cdot{ }_{3} F_{2}\left[\begin{array}{c}
-n+h, a+i s, a-i s \\
a+b, a+c
\end{array} ; 1\right]
$$

с $\operatorname{Re} a=1 / 2, \operatorname{Re} b=1 / 2, c \in \mathbb{R}$. Кроме того, по сравнению с формулой (1.4) для многочленов Хана параметр $n$ сдвигается на некоторую комплексную величину $h$.

1) Кажется, сейчас лишь разностные операторы остаются интересными. Есть 3 типа разностных операторов, связанных со следующими операторами сдвига:

(a) сдвиг на решетке $\mathbb{Z}: T f(n)=f(n+1)$,

(b) сдвиг на прямой $\mathbb{R}: T f(x)=f(x+1)$,

(c) сдвиг на прямой $\mathbb{R}$ в мнимом направлении: $T f(x)=f(x+i)$.

Кажется, в случае (b) мы получим бесконечные кратности. Так или иначе, варианты (a) и (c) интересны для наших целей. 
ЗАмЕчАниЕ. Аналогичные возмущения для нескольких более простых гипергеометрических ортогональных систем (Лагерра, Якоби, Мейкснера-Поллачека, Мейкснера) построены в $[22]^{1)}$.

1.8. Дальнейшая структура статьи. Теоремы 1.1, 1.2 доказаны в $\S 2$, теорема 1.3 - в $\S 3$. В $\S 4$ мы сводим задачу о разложении тензорного произведения представлений группы $\mathrm{SL}_{2}(\mathbb{R})$ основной серии к теореме 1.2.

\section{$\S 2$. Спектральное разложение гипергеометрического дифференциального оператора на контуре $\operatorname{Re} z=1 / 2$}

Предлагаемый ниже вывод теорем 1.1, 1.2 является прямым, но очень утомительным. Мы просто применяем теорему Г. Вейля-Титчмарша-Кодаиры к подходящему дифференциальному оператору. Сама эта теорема с большим количеством примеров разобрана в гл. XIII книги Данфорда-Шварца [5]; см. также книгу Титчмарша [31].

Для классического индексного гипергеометрического преобразования (1.3) известно несколько доказательств формулы обращения, см. $[13,23]$ и $[32,7.8 .8]$. Было бы интересно найти более короткое доказательство теоремы 1.1.

2.1. Гипергеометрический оператор. Пусть $\alpha \geqslant 0, \beta \in \mathbb{R}$. Мы рассматриваем дифференциальный оператор

$$
D:=\left(\frac{1}{4}+x^{2}\right) \frac{d^{2}}{d x^{2}}+2 x \frac{d}{d x}+\frac{(\alpha+i \beta)^{2}}{4(1 / 2+i x)}+\frac{(\alpha-i \beta)^{2}}{4(1 / 2-i x)}+\frac{1}{4} .
$$

Этот оператор формально самосопряжен в $L^{2}(\mathbb{R})$. Его резольвента $(D-\lambda)^{-1}$ ниже будет явно вычислена, и окажется, что она корректно определена при $\operatorname{Im} \lambda \neq 0$. Поэтому индексы дефекта оператора $D$ равны 0; это влечет за собой существенную самосопряженность нашего оператора. Наша цель - построить спектральное разложение оператора $D$.

Положим

$$
r(x):=\left(\frac{1}{2}+i x\right)^{-(\alpha+i \beta) / 2}\left(\frac{1}{2}-i x\right)^{-(\alpha-i \beta) / 2} .
$$

Вычисляя непосредственно дифференциальный оператор

$$
B f:=r^{-1} D(r f),
$$

получаем

$$
B=\left(\frac{1}{4}+x^{2}\right) \frac{d^{2}}{d x^{2}}+(\beta+x(2-2 \alpha)) \frac{d}{d x}+\left(\alpha-\frac{1}{2}\right)^{2} .
$$

Переходя в этом выражении к комплексной переменной

$$
z=1 / 2+i x
$$

получаем оператор

$$
A:=-z(1-z) \frac{d^{2}}{d z^{2}}-(1-\alpha-i \beta-z(2-2 \alpha)) \frac{d}{d z}-\left(\alpha-\frac{1}{2}\right)^{2}
$$

1) В [6,30] содержится попытка найти подход к векторнозначным специальным функциям с помощью сферических функций; дальнейшие ссылки см. в этой же работе. Этот подход отличен от нашего, основанного на спектральных задачах с кратными спектрами. 
Поэтому уравнение $A f=\mu^{2} f$ оказывается гипергеометрическим дифференциальным уравнением

$$
\left[z(1-z) \frac{d^{2}}{d z^{2}}+(c-(a+b+1) z) \frac{d}{d z}-a b\right] f=0
$$

C

$$
a=1 / 2-\alpha+\mu, \quad b=1 / 2-\alpha-\mu, \quad c:=1-\alpha-i \beta .
$$

Теперь мы можем использовать куммеровские ряды для решения уравнения $D f=\mu^{2} f$. Ниже мы следуем стандартным обозначениям $u_{1}, \ldots, u_{6}$ из [7] для куммеровских решений стандартного гипергеометрического уравнения (2.3) и используем явные формулы (2.9.1)-(2.9.24) из [7] для их разложений в ряды.

2.2. Базисы в пространстве решений гипергеометрического уравнения. Мы будем использовать четыре базиса в пространстве решений уравнения $\left(D-\mu^{2}\right) f=0$.

Базис $S_{1}, S_{2}$. Выписывая решения Куммера $u_{1}, u_{5}$ стандартного гипергеометрического уравнения (2.3), мы получаем следующую пару решений $S_{1}, S_{2}$ уравнения (2.1):

$$
\begin{aligned}
S_{1}(\alpha, \beta ; \mu ; x):= & \left(\frac{1}{2}+i x\right)^{-(\alpha+i \beta) / 2}\left(\frac{1}{2}-i x\right)^{(\alpha+i \beta) / 2-\mu-1 / 2} \\
& \times{ }_{2} F_{1}\left[\begin{array}{c}
1 / 2-\alpha+\mu, 1 / 2-i \beta+\mu \\
1-\alpha-i \beta
\end{array} \frac{i x+1 / 2}{i x-1 / 2}\right], \\
S_{2}(\alpha, \beta ; \mu ; x)= & S_{1}(-\alpha,-\beta ; \mu ; x) .
\end{aligned}
$$

Гипергеометрический ряд в (2.4) абсолютно сходится при $\operatorname{Im} x>0$, или, что то же самое, при $\operatorname{Re} z<1 / 2$. На прямой $\operatorname{Im} x=0$ он сходится условно. Он, однако, аналитически продолжается через эту прямую, и удобнее считать, что мы рассматриваем это аналитическое продолжение.

Далее предположим, что при $x=i y$ с $-1 / 2<y<1 / 2$ (или, что то же самое, при $0<z<1$ )

$$
\left(\frac{1}{2}+i x\right)^{\lambda}:=e^{\lambda \ln (1 / 2+i x)}, \quad\left(\frac{1}{2}-i x\right)^{\nu}:=e^{\nu \ln (1 / 2-i x)} .
$$

Теперь мы можем считать, что наши решения $S_{1}, S_{2}$ определены в области $\{\operatorname{Re} z \leqslant 1 / 2\} \backslash[-\infty, 0)$.

Решения $S_{1}, S_{2}$ имеют асимптотики

$$
S_{1}(x) \sim\left(\frac{1}{2}+i x\right)^{(\alpha+i \beta) / 2}, S_{2}(x) \sim\left(\frac{1}{2}+i x\right)^{-(\alpha+i \beta) / 2} \quad \text { при } x \rightarrow i / 2 .
$$

Мы также можем задать $S_{1}$ формулой

$$
\left(\frac{1}{2}+i x\right)^{-(\alpha+i \beta) / 2}\left(\frac{1}{2}-i x\right)^{-(\alpha-i \beta) / 2}{ }_{2} F_{1}\left[\begin{array}{c}
1 / 2-\alpha+\mu, 1 / 2-\alpha-\mu \\
1-\alpha-i \beta
\end{array} ; x+1 / 2\right] .
$$

Здесь гипергеометрический ряд сходится при $|1 / 2-i x|<1 / 2$, т. е. явной формулы на всей прямой $\operatorname{Im} x=0$ мы, вообще говоря, не получаем.

Отметим, что

$$
S_{1}(\alpha, \beta ;-\mu ; x)=S_{1}(\alpha, \beta ; \mu ; x)
$$


Базис $T_{1}, T_{2}$. Выписывая пару куммеровских решений $u_{2}, u_{6}$, мы получаем следующую пару решений $T_{1}, T_{2}$ уравнения $\left(D-\mu^{2}\right) f=0$ :

$$
T_{1,2}(\alpha, \beta ; \mu ; x):=S_{1,2}(\alpha,-\beta ; \mu ;-x) .
$$

Эти решения определены в области $\{\operatorname{Re} z \geqslant 1 / 2\} \backslash[1, \infty)$.

Базисы $V_{-}, V_{+} u W_{-}, W_{+}$. Куммеровские решения $u_{3}, u_{4}$ определены вне лунки $|z|<1,|z-1|<1$; эта лунка, в частности, содержит отрезок $-\sqrt{3} / 2<$ $x<\sqrt{3} / 2$. Мы определяем решение $V_{-}$уравнения $\left(D-\mu^{2}\right) f=0$ как решение, которое при $x>\sqrt{3} / 2$ задается формулой

$$
\begin{aligned}
V_{-}(\alpha, \beta ; \mu ; x)=e^{(-1 / 2+\alpha+i \beta-\mu) \pi i / 2}\left(\frac{1}{2}+i x\right)^{-(\alpha+i \beta) / 2}\left(\frac{1}{2}-i x\right)^{-1 / 2+(\alpha+i \beta) / 2-\mu} \\
\times{ }_{2} F_{1}\left[\begin{array}{c}
1 / 2-\alpha+\mu, 1 / 2-i \beta+\mu \\
1+2 \mu
\end{array} \frac{1}{1 / 2-i x}\right] . \quad(2.7)
\end{aligned}
$$

Далее, мы полагаем

$$
V_{+}(\alpha, \beta ; \mu ; x)=V_{-}(\alpha, \beta ;-\mu ; x) .
$$

Асимптотика $V_{ \pm}$при $x \rightarrow+\infty$ имеет вид

$$
V_{ \pm} \sim x^{1 / 2 \pm \mu}, \quad x \rightarrow+\infty .
$$

Решения $W_{ \pm}$мы определяем из условия

$$
W_{ \pm} \sim x^{1 / 2 \pm \mu}, \quad x \rightarrow-\infty .
$$

Чтобы получить формулу для $W_{-}$при $x<\sqrt{3} / 2$, мы должны сменить знак в показателе экспоненциальной функции в $(2.7)$. Далее, $W_{+}(\alpha, \beta ; \mu ; x)=$ $W_{-}(\alpha, \beta ;-\mu ; x)$.

2.3. Матрицы перехода. Определим константы

$$
\begin{aligned}
C(\alpha, \beta ; \mu) & :=\frac{\Gamma(\alpha+i \beta) \Gamma(1+2 \mu)}{\Gamma(1 / 2+\alpha+\mu) \Gamma(1 / 2+i \beta+\mu)}, \\
\chi(\alpha, \beta ; \mu) & :=e^{-(1 / 2+\alpha+i \beta-\mu) \pi i / 2} .
\end{aligned}
$$

В этих обозначениях (мы используем $[7,(2.9 .37),(2.9 .39)])$

$$
\begin{aligned}
V_{-}(\alpha, \beta ; \mu ; x)=C & (\alpha, \beta ; \mu) \chi(\alpha, \beta ; \mu) S_{1}(\alpha, \beta ; \mu ; x) \\
& +C(-\alpha,-\beta ; \mu) \chi(-\alpha,-\beta ; \mu) S_{2}(\alpha, \beta ; \mu ; x), \\
V_{+}(\alpha, \beta ; \mu ; x)=C & (\alpha, \beta ;-\mu) \chi(\alpha, \beta ;-\mu) S_{1}(\alpha, \beta ; \mu ; x) \\
& +C(-\alpha,-\beta ;-\mu) \chi(-\alpha,-\beta ;-\mu) S_{2}(\alpha, \beta ; \mu ; x) .
\end{aligned}
$$

Формулы, выражающие $W_{-}, W_{+}$через $S_{1}, S_{2}$, получаются из $(2.12),(2.13)$ заменой $\chi \mapsto \chi^{-1}$.

Нам также нужно будет выразить $W_{ \pm}$через $T_{1}, T_{2}$. Аналогично, используя $[7,(2.9 .38),(2.9 .40)]$, получаем

$$
\begin{aligned}
W_{-}(\alpha, \beta ; \mu ; x)=- & C(\alpha,-\beta ; \mu) \chi^{-1}(-\alpha, \beta ;-\mu) T_{1}(\alpha, \beta ; \mu ; x) \\
& -C(-\alpha, \beta ; \mu) \chi^{-1}(\alpha,-\beta ;-\mu) T_{2}(\alpha, \beta ; \mu ; x) ;
\end{aligned}
$$

в формуле для $W_{+}$нужно сменить знак перед $\mu$ в $C(\alpha, \beta ; \mu)$ и в $\chi(\alpha, \beta ; \mu)$. 
2.4. Вычисление вронскианов. Обозначим через $\mathbf{w r}(P, Q):=\operatorname{det}\left(\begin{array}{cc}P & Q \\ P^{\prime} & Q^{\prime}\end{array}\right)$ вронскиан двух каких-либо решений $P, Q$ уравнения $\left(D-\mu^{2}\right) f=0$. Это выражение должно иметь вид

$$
\mathbf{w r}(P, Q)=\frac{\sigma(P, Q)}{1 / 4+x^{2}}
$$

где $\sigma(P, Q)$ - постоянная, см. [10, I.17.1].

Величина $\sigma\left(S_{1}, S_{2}\right)$ легко находится из асимптотик $(2.5)$ :

$$
\sigma\left(S_{1}, S_{2}\right)=i(\alpha+i \beta)
$$

Ниже нам будет нужно знать $\sigma\left(V_{-}, W_{-}\right)$. Определитель $\Delta$ матрицы перехода от базиса $\left(S_{1}, S_{2}\right)$ к базису $\left(V_{-}, W_{-}\right)$легко считается, и это дает

$$
\sigma\left(V_{-}, W_{-}\right)=\Delta \cdot \sigma\left(S_{1}, S_{2}\right)=\frac{2 \pi i C(\alpha, \beta ; \mu) C(-\alpha,-\beta ; \mu)}{\Gamma(\alpha+i \beta) \Gamma(-\alpha-i \beta)} .
$$

2.5. Ядро резольвенты. Теперь мы готовы выписать ядро $K(x, y ; \lambda)$ peзольвенты

$$
R(\lambda):=(D-\lambda)^{-1}
$$

оператора $D$,

$$
R(\lambda) f(x)=\int_{-\infty}^{\infty} K(x, y ; \lambda) f(y) d y
$$

Пусть $\lambda \in \mathbb{C} \backslash(-\infty, 0)$. Тогда решение $V_{-}$лежит в $L^{2}(0,+\infty)$, а $W_{-}-$в $L^{2}(0,-\infty)$, см. (2.8), (2.9). Следовательно (см. [5, ХІІІ.3.6]),

$$
K(x, y ; \lambda)= \begin{cases}\frac{V_{-}(x) W_{-}(y)}{\sigma\left(V_{-}, W_{-}\right)} & \text {при } y<x, \\ \frac{V_{-}(y) W_{-}(x)}{\sigma\left(V_{-}, W_{-}\right)} & \text {при } x<y ;\end{cases}
$$

величина $\sigma\left(V_{-}, W_{-}\right)$вычислена выше (2.15).

Мы хотим написать разложение дифференциального оператора $R$ по собственным функциям. Согласно рецепту, восходящему к работе Вейля [33] (см. [31] и обстоятельное изложение у Данфорда-Шварца [5, XIII.5.18]), мы должны вычислить скачок резольвенты на вещественной оси

$$
\frac{1}{2 \pi i} \lim _{\varepsilon \rightarrow 0} \int_{-\infty}^{\infty}(R(\lambda+i \varepsilon)-R(\lambda-i \varepsilon)) d \lambda
$$

и представить его в виде

$$
L f(x)=\sum_{i=1,2} \sum_{j=1.2} \int_{-\infty}^{\infty}\left(\int_{-\infty}^{\infty} \sigma_{i}(x, \lambda) \overline{\left.\sigma_{j}(y, \lambda)\right)} f(y) d \mu_{i j}(\lambda)\right) d y
$$

для некоторых решений $\sigma_{1}, \sigma_{2}$ уравнения $D f=\lambda f$. Здесь $\mu_{i j}(\lambda)$ - меры (комплекснозначные) на $\mathbb{R}$. Тогда $\mu_{i j}$ - спектральная мера. Точнее, оператор

$$
f \mapsto\left(\int_{-\infty}^{\infty} f(x) \overline{\sigma_{1}(x)} d x, \int_{-\infty}^{\infty} f(x) \overline{\sigma_{2}(x)} d x\right)
$$


является унитарным оператором из $L^{2}(\mathbb{R})$ в пространство $\mathbb{C}^{2}$-значных функций $\left(\varphi_{1}(\lambda), \varphi_{2}(\lambda)\right)$ со скалярным произведением

$$
\left\langle\left(\varphi_{1}(\lambda), \varphi_{1}(\lambda),\left(\psi_{1}(\lambda), \psi_{1}(\lambda)\right\rangle=\sum_{i=1,2} \sum_{i=1,2} \int_{-\infty}^{\infty} \varphi_{i}(\lambda) \overline{\varphi_{j}(\lambda)} d \mu_{i j}(\lambda)\right.\right.
$$

(в частности, правая часть равенства - действительно положительно определенное скалярное произведение).

2.6. Формула для резольвенты. Для вычисления скачка резольвенты при $\lambda \geqslant 0$ и при $\lambda \leqslant 0$ нам понадобятся две явные формулы для ее ядра $K(x, y ; \lambda)$.

Выражая $V_{-}$через $S_{1}, S_{2}$, а $W_{-}$через $T_{1}, T_{2}$, получаем следующую формулу:

$$
\begin{aligned}
K(x, y ; \lambda)=\frac{-1}{2 \pi} \Gamma(\alpha+i \beta) \Gamma(- & \alpha-i \beta)\left[\frac{C(\alpha,-\beta ; \sqrt{\lambda})}{C(-\alpha,-\beta ; \sqrt{\lambda})} e^{(-1 / 2+\alpha-\sqrt{\lambda}) \pi i} S_{1}(x) T_{1}(y)\right. \\
& +\frac{C(-\alpha, \beta ; \sqrt{\lambda})}{C(-\alpha,-\beta ; \sqrt{\lambda})} e^{(-1 / 2+i \beta-\sqrt{\lambda}) \pi i} S_{1}(x) T_{2}(y) \\
& +\frac{C(\alpha,-\beta ; \sqrt{\lambda})}{C(\alpha, \beta ; \sqrt{\lambda})} e^{(-1 / 2-i \beta-\sqrt{\lambda}) \pi i} S_{2}(x) T_{1}(y) \\
& \left.\left.+\frac{C(-\alpha, \beta ; \sqrt{\lambda})}{C(\alpha, \beta ; \sqrt{\lambda})} e^{(-1 / 2-\alpha-\sqrt{\lambda}) \pi i} S_{2}(x) T_{2}(y)\right], \quad 2.18\right)
\end{aligned}
$$

где $C(\cdots)$ задано формулой (2.10).

Если мы выразим $V_{-}, W_{-}$через $S_{1}, S_{2}$, то получим

$$
\begin{aligned}
& K(x, y ; \lambda)=\frac{\Gamma(\alpha+i \beta) \Gamma(-\alpha-i \beta)}{2 \pi C(\alpha, \beta ; \sqrt{\lambda}) C(-\alpha,-\beta ; \sqrt{\lambda})} \\
& \left.\left.\left.\quad \times[C(\alpha, \beta ; \sqrt{\lambda})) \chi(\alpha, \beta ; \sqrt{\lambda}) S_{1}(x)+C(-\alpha,-\beta ; \sqrt{\lambda})\right) \chi(-\alpha,-\beta ; \sqrt{\lambda})\right) S_{2}(x)\right] \\
& \left.\left.\left.\quad \times[C(\alpha, \beta ; \sqrt{\lambda})) \chi^{-1}(\alpha, \beta ; \sqrt{\lambda}) S_{1}(x)+C(-\alpha,-\beta ; \sqrt{\lambda})\right) \chi^{-1}(-\alpha,-\beta ; \sqrt{\lambda})\right) S_{2}(x)\right],
\end{aligned}
$$

где $\chi$ задано формулой (2.11).

Эти два выражения являются мероморфными в плоскости $\lambda \in \mathbb{C}$, разрезанной по отрицательной полуоси $\lambda<0$.

2.7. Скачок резольвенты при $\boldsymbol{\lambda}>0$. Вычисление величины

$$
L^{[0, \infty)}:=\frac{1}{2 \pi i} \lim _{\varepsilon \rightarrow 0} \int_{0}^{\infty}(R(\lambda+i \varepsilon)-R(\lambda-i \varepsilon)) d \lambda
$$

сводится к вычислению вычетов. Выражение (2.19) имеет полюсы первого порядка в точках $\lambda=(\alpha-k-1 / 2)^{2}$ при целых $k$, удовлетворяющих условиям $0 \leqslant k \leqslant \alpha-1 / 2$. При этом лишь коэффициент при $S_{1}(x) S_{1}(y)$ действительно имеет ненулевые вычеты, три других коэффициента голоморфны в данных точках. 
В итоге интегральный оператор (2.20) оказывается равным

$$
\begin{aligned}
L^{[0, \infty)} f(x)=\frac{1}{2 \pi} & \Gamma(\alpha+i \beta) \Gamma(\alpha-i \beta) \sum_{0 \leqslant k<\alpha-1 / 2} \frac{(2 \alpha-2 k-1)(1-\alpha-i \beta)_{k}}{\Gamma(2 \alpha-k)(1-\alpha+i \beta)_{k} k !} \\
& \times \int_{-\infty}^{\infty} S_{1}(\alpha, \beta ; \alpha-k-1 / 2 ; x) S_{1}(\alpha, \beta ; \alpha-k-1 / 2 ; y) f(y) d y .
\end{aligned}
$$

Записывая функцию $S_{1}$ в форме (2.6), мы получаем ее представление через многочлен Романовского. С помощью тождества (10.8.16) из [7] для многочленов Якоби мы приводим это выражение к виду

$$
\begin{array}{r}
\frac{1}{2 \pi} \Gamma(\alpha+i \beta) \Gamma(\alpha-i \beta) \sum_{0 \leqslant k<\alpha-1 / 2} \frac{2 \alpha-2 k-1}{\Gamma(2 \alpha-k) k !} S_{1}(\alpha, \beta ; \alpha-k-1 / 2 ; x) \\
\times \int_{-\infty}^{\infty} \overline{S_{1}(\alpha, \beta ; \alpha-k-1 / 2 ; y)} f(y) d y
\end{array}
$$

и это дает искомое представление.

2.8. Скачок резольвенты при $\boldsymbol{\lambda}<0$. Мы должны вычислить

$$
K(x, y ; \sqrt{\lambda})-K(x, y ;-\sqrt{\lambda}),
$$

считая, что $\lambda$ - отрицательное число, a $\operatorname{Im} \sqrt{\lambda}>0$.

Мы для определенности проведем вычисление коэффициента при $S_{1}(x) T_{1}(y)$. В трех других случаях вычисление почти идентично ${ }^{1)}$.

Итак (см. (2.18)), мы хотим найти

$$
\begin{aligned}
& \frac{1}{-2 \pi} \Gamma(\alpha+i \beta) \Gamma(-\alpha-i \beta) \\
& \quad \times\left\{\frac{C(\alpha,-\beta ; \sqrt{\lambda})}{C(-\alpha,-\beta ; \sqrt{\lambda})} e^{-(1 / 2-\alpha+\sqrt{\lambda}) \pi i}-\frac{C(\alpha,-\beta ;-\sqrt{\lambda})}{C(-\alpha,-\beta ;-\sqrt{\lambda})} e^{-(1 / 2-\alpha-\sqrt{\lambda}) \pi i}\right\} .
\end{aligned}
$$

После непосредственных сокращений получаем

$$
\begin{aligned}
& \frac{1}{-2 \pi} \Gamma(\alpha+i \beta) \Gamma(\alpha-i \beta) \\
& \quad \times\left\{\frac{\Gamma(1 / 2-\alpha+\sqrt{\lambda})}{\Gamma(1 / 2+\alpha+\sqrt{\lambda})} e^{-(1 / 2-\alpha+\sqrt{\lambda}) \pi i}+\frac{\Gamma(1 / 2-\alpha-\sqrt{\lambda})}{\Gamma(1 / 2+\alpha-\sqrt{\lambda})} e^{-(1 / 2-\alpha-\sqrt{\lambda}) \pi i}\right\} .
\end{aligned}
$$

Мы преобразуем это к виду

$$
\begin{aligned}
& \frac{1}{-2 \pi} \Gamma(\alpha+i \beta) \Gamma(\alpha-i \beta) \Gamma(1 / 2-\alpha+\sqrt{\lambda}) \Gamma(1 / 2-\alpha-\sqrt{\lambda}) \\
& \times\left\{\frac{e^{-(1 / 2-\alpha+\sqrt{\lambda}) \pi i}}{\Gamma(1 / 2+\alpha+\sqrt{\lambda}) \Gamma(1 / 2-\alpha-\sqrt{\lambda})}-\frac{e^{-(1 / 2-\alpha-\sqrt{\lambda}) \pi i}}{\Gamma(1 / 2+\alpha-\sqrt{\lambda}) \Gamma(1 / 2-\alpha+\sqrt{\lambda})}\right\} .
\end{aligned}
$$

Теперь мы будем преобразовывать лишь выражение в фигурных скобках:

$$
\pi\{\cdots\}=\cos (\pi(\alpha+\sqrt{\lambda})) e^{-(1 / 2-\alpha+\sqrt{\lambda}) \pi i}-\cos (\pi(\alpha-\sqrt{\lambda})) e^{-(1 / 2-\alpha-\sqrt{\lambda}) \pi i} .
$$

1)Это обеспечивается естественными симметриями $(\alpha, \beta) \sim(-\alpha,-\beta) \sim(i \beta,-i \alpha)$ в уравнении (2.1). 
Далее мы применяем формулу Эйлера для cos, приводим подобные члены в линейной комбинации экспонент и еще раз применяем формулу Эйлера. Получаем

$$
i \sin (2 \sqrt{\lambda} \pi i)
$$

и это завершает вычисление коэффициента при $S_{1}(x) T_{1}(y)$.

После всего этого мы получим для скачка резольвенты $L^{(-\infty, 0]}$ на полуоси $(-\infty, 0]$ выражение вида

$$
L^{(-\infty, 0)} f(x)=\sum_{i=1,2} \sum_{j=1,2} \int_{-\infty}^{0} d \lambda\left\{\theta_{i j}(\lambda) \int_{-\infty}^{\infty} S_{i}(x) T_{j}(y) f(y) d y\right\},
$$

где $\theta_{i j}$ - явные произведения Г-функций.

Далее мы замечаем, что при $\lambda<0$ и при $y \in \mathbb{R}$ выполнено равенство $T_{j}(y)=\overline{S_{j}(y)}$. Мы получаем выражение вида (2.17), и это завершает вычисление спектрального разложения дифференциального оператора (2.1).

Параметр $s$ из разд. 1.4 есть $\sqrt{\lambda}$.

\section{§3. Построение базисов}

3.1. Один базис в $\boldsymbol{L}^{2}(\mathbb{R})$. Фиксируем вещественные параметры $p$ и $q$.

Лемма 3.1. Система функиий

$$
r_{p, q}^{(n)}(x):=\left(\frac{1}{2}+i x\right)^{-1 / 2-n-(p+i q) / 2}\left(\frac{1}{2}-i x\right)^{-1 / 2+n+(p-i q) / 2},
$$

где $n$ пробегает целые числа, образует ортонормированный базис в $L^{2}(\mathbb{R}, d x / 2 \pi)$.

ДокАЗАТЕЛЬСтво. Перейдем к новой переменной $\psi \in[0,2 \pi]$ по формуле

$$
e^{i \psi}=\frac{1 / 2+i x}{1 / 2-i x}, \quad d \psi=\frac{d x}{1 / 4+x^{2}} .
$$

Наша система функций переходит в $e^{-i n \psi} \cdot e^{-i p \psi / 2}(2 \cos \psi / 2)^{i q}$. Мы получили стандартную ортогональную систему $e^{-i n \psi}$ с точностью до умножения на функцию, по модулю равную 1.

Отметим, что ортогональность нашей системы легко может быть проверена непосредственно (скалярные произведения легко вычисляются с помощью вычетов).

3.2. Построение базисов. Базисы (1.13) получаются применением нашего интегрального преобразования к ортогональной системе (3.1).

Объясним, как провести вычисление. Мы должны найти

$$
\int_{-\infty}^{\infty} r_{p, q}^{(n)}(x) \overline{Q_{1}(\alpha, \beta ; x ; s)} d x
$$

где функция $Q_{1}$ задана формулой (1.6). Для этого мы разлагаем ${ }_{2} F_{1}$ из формулы (1.6) в гипергеометрический ряд по степеням $(i x+1 / 2) /(i x-1 / 2)$ и интегрируем его почленно с помощью бета-интеграла Коши

$$
\int_{-\infty}^{\infty} \frac{d x}{(1 / 2+i x)^{\sigma}(1 / 2-i x)^{\tau}}=\frac{2 \pi \Gamma(\sigma+\tau-1)}{\Gamma(\sigma) \Gamma(\tau)}
$$


(см. [25, т. 1, (2.2.6.31)] или [1, ch. 1, ех. 13]). В итоге для (3.2) получается выражение

$$
\begin{gathered}
2 \pi \Gamma(1 / 2+i q-i s) \\
\Gamma(\alpha-i \beta) \Gamma((p+i q-\alpha+i \beta) / 2+n+1-i s) \Gamma((-p+i q+\alpha-i \beta+1) / 2-n) \\
\times{ }_{3} F_{2}\left[\begin{array}{c}
1 / 2-\alpha-i s, 1 / 2+i \beta-i s, 1 / 2+(p-i q-\alpha+i \beta) / 2+n \\
1-\alpha+i \beta,(p+i q-\alpha+i \beta) / 2+n+1-i s
\end{array}\right] .
\end{gathered}
$$

Эта формула является вариантом окончательного ответа; более симметричная форма (1.13) получается с помощью преобразования Томе, см. [1, corollary 3.3.6] и $[25$, т. $3,(7.4 .4 .2)]$ :

$$
{ }_{3} F_{2}\left[\begin{array}{c}
a, b, c \\
d, e
\end{array} ; 1\right]=\frac{\Gamma(d) \Gamma(e) \Gamma(r)}{\Gamma(a) \Gamma(b+r) \Gamma(c+r)}{ }_{3} F_{2}\left[\begin{array}{c}
d-a, e-a, r \\
b+r, c+r
\end{array} ; 1\right],
$$

где $r=d+e-a-b-c$.

\section{§4. Приложение к тензорным произведениям унитарных представлений $\mathrm{SL}_{2}(\mathbb{R})$}

4.1. Модели представлений. Мы реализуем группу $\mathrm{SL}_{2}(\mathbb{R})$ как группу комплексных $2 \times 2$-матриц вида $g=\left(\begin{array}{cc}a & b \\ \bar{b} & \bar{a}\end{array}\right)$, таких, что $|a|^{2}-|b|^{2}=1$. Эта группа действует на окружности $S^{1}:|z|=1$ мёбиусовскими преобразованиями $z \mapsto(a z+b) /(\bar{b} z+\bar{a})$.

Для фиксированных $\theta, \tau \in \mathbb{C}$ определим представление $T_{\theta, \tau}$ группы $\mathrm{SL}_{2}(\mathbb{R})$ в пространстве $C^{\infty}\left(S^{1}\right)$ по формуле

$$
T_{\theta, \tau}(g) f(z)=f((a z+b) /(\bar{b} z+\bar{a}))(\bar{b} z+\bar{a})^{-\theta}\left(b z^{-1}+a\right)^{-\tau} .
$$

Объясним, как понимать степени комплексных чисел в этой формуле, если $\theta, \tau \notin \mathbb{Z}$. У нас $|a|>|b|$; поэтому $(\bar{b} z+\bar{a})$ не обращается в нуль внутри круга $|z| \leqslant 1$. Следовательно, функция $\ln (\bar{b} z+\bar{a})$ имеет счетное число гладких ветвей в круге и, в частности, на окружности $S^{1}$. Эти ветви отличаются друг от друга на аддитивную константу вида $2 \pi k i$, где $k \in \mathbb{Z}$. Далее, при $|z|=1$ выполнено равенство $b z^{-1}+a=\overline{(\bar{b} z+\bar{a})}$. Поэтому, фиксировав ветвь логарифма, мы однозначно определяем выражение (4.1).

Следовательно, при фиксированной матрице $g$ формула (4.1) задает счетное (иногда конечное) число непрерывных операторов в $C^{\infty}\left(S^{1}\right)$, отличающихся постоянными множителями. В итоге мы получаем проективное представление группы $\mathrm{SL}_{2}(\mathbb{R})$ (или линейное представление ее универсальной накрывающей группы $\left.\mathrm{SL}_{2}(\mathbb{R})^{\sim}\right)$.

Генераторы алгебры Ли $s l_{2}$ действуют дифференциальными операторами

$$
L_{0}=2 z \frac{d}{d z}+\theta-\tau, \quad L_{1}=z^{2} \frac{d}{d z}+\theta z, \quad L_{-1}=\frac{d}{d z}-\tau z^{-1} .
$$

Если $\operatorname{Re}(\theta+\tau)=1, \operatorname{Im} \theta=\operatorname{Im} \tau$, то представления $T_{\theta, \tau}$ унитарны в $L^{2}\left(S^{1}\right)$; такие представления называются представлениями основной серии. Отметим, что представления $T_{\theta+n, \tau-n}$ и $T_{\theta, \tau}$ эквивалентны (сплетающий их оператор это умножение на $z^{n}$ ). 
4.2. Тензорные произведения и оператор Лапласа на торе. Рассмотрим тензорное произведение представлений $T_{\theta, \tau}$ и $T_{\widetilde{\theta}}, \widetilde{\tau}$. Мы реализуем его естественным образом в функциях на торе $|z|=|u|=1$. Рассмотрим оператор Лапласа

$$
\Delta:=\frac{1}{4}\left(L_{0}(z)+L_{0}(u)\right)^{2}+\frac{1}{2}\left(L_{0}(z)+L_{0}(u)\right)-\left(L_{-1}(z)+L_{-1}(u)\right)\left(L_{1}(z)+L_{1}(u)\right) .
$$

Здесь $L_{j}(z)$ - это операторы $(4.2)$, а $L_{j}(u)$ - такие же операторы, только $z$ заменено на $u$, а $\theta, \tau-$ на $\widetilde{\theta}, \widetilde{\tau}$. Оператор $\Delta$ коммутирует со всеми операторами $L_{j}(z)+L_{j}(u)$.

Прямое вычисление дает

$$
\begin{aligned}
\Delta=-(z & -u)^{2} \frac{\partial^{2}}{\partial z \partial u}+\frac{1}{u}(\widetilde{\theta} u+\widetilde{\tau} z)(z-u) \frac{\partial}{\partial z}+\frac{1}{z}(\theta z+\tau u)(u-z) \frac{\partial}{\partial u} \\
& +\frac{1}{z u}(\widetilde{\theta} u+\widetilde{\tau} z)(\theta z+\tau u)+\frac{1}{4}(\theta-\widetilde{\theta}+\tau-\widetilde{\tau})^{2}+\frac{1}{4}(\theta+\tau+\widetilde{\theta}+\widetilde{\tau}) .
\end{aligned}
$$

Вычисление меры Планшереля для тензорного произведения сводится к спектральному разложению оператора $\Delta$ (см., например, $[26,19])$.

4.3. Сведе́ние к одномерному оператору. Рассмотрим функции на торе вида $f(z, u)=g(z / u)$. Такие функции являются собственными относительно оператора $L_{0}(z)+L_{0}(u)$,

$$
\left(L_{0}(z)+L_{0}(u)\right) g(z / u)=(\theta+\widetilde{\theta}-\tau-\widetilde{\tau}) g(z / u) .
$$

На функциях $g(w)$ мы получаем оператор

$$
\begin{aligned}
w(1-w)^{2} & \frac{d^{2}}{d w^{2}}+(w-1)(\tau+\widetilde{\theta}-1+(w-1)(\widetilde{\tau}+\theta)) \frac{d}{d w} \\
& +\widetilde{\tau} \theta(w-1)+\tau \widetilde{\theta}\left(\frac{1}{w}-1\right)+\frac{1}{4}(\tau+\theta+\widetilde{\tau}+\widetilde{\theta})^{2}-\frac{1}{2}(\tau+\theta+\widetilde{\tau}+\widetilde{\theta}) .
\end{aligned}
$$

Переменная $w$ пробегает контур $|w|=1$. Далее мы переходим к функции

$$
h(t):=\left(\frac{1}{2}+i t\right)^{(\tau+\widetilde{\theta}) / 2}\left(\frac{1}{2}-i t\right)^{(\widetilde{\tau}+\theta) / 2} g\left(\frac{i t+1 / 2}{i t-1 / 2}\right)
$$

и получаем оператор

$$
D:=\left(\frac{1}{4}+t^{2}\right) \frac{d^{2}}{d t^{2}}+2 t \frac{d}{d t}+\frac{1}{4}\left(\frac{(\theta-\widetilde{\tau})^{2}}{1 / 2-i t}+\frac{(\widetilde{\theta}-\tau)^{2}}{1 / 2+i t}\right)
$$

на прямой $\mathbb{R}$. Наш оператор имеет вид $(2.1)$ с, вообще говоря, комплексными $\alpha$ и $\beta$. Если наши начальные представления $T_{\theta, \tau}, T_{\widetilde{\theta}, \widetilde{\tau}}$ лежали в основной серии, то мы получаем оператор вида (2.1) с вещественными $\alpha, \beta$.

Итак, мы разобрали оператор Лапласа, ограниченный на собственное подпространство (4.4) оператора $L_{0}(z)+L_{0}(u)$. Но преобразование $\theta \mapsto \theta-n$, $\tau \mapsto \tau+n$ фактически не меняет задачу, так как соответствующие представления $T_{\theta+n, \tau-n}$ и $T_{\theta, \tau}$ эквивалентны; таким образом, мы знаем спектральное разложение оператора Лапласа на любом собственном подпространстве оператора $L_{0}(z)+L_{0}(u)$. 
ЗАмЕчАниЕ. Отметим, что любой оператор вида $(2.1)$ с $\alpha, \beta \in \mathbb{R}$ возникает только что описанным естественным способом.

\section{ЛитЕРАТУРА}

1. Andrews G. E., Askey R., Roy R. Special functions. Cambridge Univ. Press, 1999; готовится русский перевод.

2. Askey $R$. An integral of Ramanujan and orthogonal polynomials. J. Indian Math. Soc. (N.S.), 51, 27-36 (1987).

3. Borodin A., Olshanski $G$. Harmonic analysis on the infinite-dimensional unitary group and determinantal point processes. Preprint, http://arXiv.org/abs/math.RT/ 0109194.

4. Borodin A., Olshanski G. Random partitions and the Gamma kernel. Preprint, http: //arXiv.org/abs/math-ph/0305043.

5. Dunford N., Schwartz J. T. Linear operators. Vol. 2. Wiley \& Sons, 1963. [Имеется перевод: Данфорд М., Швари, Дж. Линейные операторы. Т. 2. Мир, М., 1966.]

6. Grunbaum F. A., Pacharoni I., Tirao J. Matrix valued spherical functions associated to the complex projective plane. J. Funct. Anal., 188, No. 2, 350-441 (2002).

7. Erdelyi A., Magnus W., Oberhetinger F., Tricomi F. Higher transcendental functions. Vol. 1, 2, McGray-Hill, 1953. [Имеется перевод: Эрдеи А., Магнус В., Оберхетингер $\Phi .$, Трикоми $\Phi$. Высшие трансцендентные функции. Наука, М., 1966.]

8. Flensted-Jensen M., Koornwinder T. The convolution structure for Jacobi function expansions. Ark. Math., 11, 245-262 (1973).

9. Groenevelt $W$. The Wilson function transform. Preprint, http://xxx.lanl.gov/abs/ math.CA/0306424.

10. Kamke E. Differentialgleichungen. I. Gevöhnliche Differentialgleichungen. 6 ed., Leipzig, 1959. [Имеется перевод: Камке Э. Справочник по обыкновенным дифференциальным уравнениям. Наука, М., 1971.]

11. Koekoek R., Swarttouw R. F. The Askey-scheme of hypergeometric orthogonal polynomials and its $q$-analogue. Delft University of Technology, Faculty of Technical Mathematics and Informatics, Report no. 94-05, 1994, http://aw.twi.tudelft.nl/ koekoek/askey.html.

12. Koornwinder T. H. A new proof of a Paley-Wiener theorem for Jacobi transform. Ark. Math., 13, 145-159 (1975).

13. Koornwinder T. H. Jacobi functions and analysis on noncompact symmetric spaces. In: Special functions: group theoretical aspects and applications (Askey R., Koornwinder T., eds.). Reidel, Dodrecht-Boston, 1984, pp. 1-85.

14. Lesky $P$. A. Endliche und unendliche Systeme von kontinuierlichen klassischen Orthogonalpolynomen. Z. Angew. Math. Mech., 76, No. 3, 181-184 (1996).

15. Lesky P. A. Unendliche und endliche Orthogonalsysteme von continuous Hahnpolynomen. Results Math., 31, No. 1-2, 127-135 (1997).

16. Lesky P. A., Waibel B.-M. Orthogonalitat von Racahpolynomen und Wilsonpolynomen. Results Math., 35, No. 1-2, 119-133 (1999).

17. Молчанов $B$. $\Phi$. Тензорные произведения унитарных представлений трехмерной группы Лоренца. Изв. АН СССР, сер. мат., 43, 860-891 (1979).

18. Молчанов $B$. Ф. Формула Планшереля для псевдоримановых симметрических пространств ранга 1. ДАН СССР, 290, №3, 545-549 (1986).

19. Молчанов $B$. Ф. Гармонический анализ на однородных пространствах. Итоги науки и техники. Современные проблемы математики. Фундаментальные направления, Т. 59. Некоммутативный гармонический анализ-II. ВИНИТИ, М., 1990, с. 5144.

20. Неретин Ю. А. Индексное гипергеометрическое преобразование и имитация анализа ядер Березина на гиперболических пространствах. Матем. сб., 192, №3, 83114 (2001); Препринтный вариант: http://arXiv.org/abs/math/0104035. 
21. Неретин Ю. А. Бета-интегралы и конечные ортогональные системы многочленов Вильсона. Матем. сб., 193, No. 7, 131-148 (2002).

22. Neretin $Y u$. A. Perturbations of some classical hypergeometric orthogonal systems. Preprint, http:/!/arXiv.org/abs/math/a0309445.

23. Олевский M. H. О представлении произвольной функции в виде интеграла с ядром, являющимся гипергеометрической функцией. ДАН СССР, 69, №1, 11-14 (1949).

24. Peetre J. Correspondence principle for the quantized annulus, Romanovski polynomials, and Morse potential. J. Funct. Anal., 117, No. 2, 377-400 (1993).

25. Прудников А. М., Брычков Ю. А., Маричев О. И. Интегралы и ряды. Т. 1-3. Наука, M., 1981, 1983, 1986.

26. Pukanszky L. On the Kronecker products of irreducible unitary representations of the $2 \times 2$ real unimodular group. Trans. Amer. Math. Soc., 100, 116-152 (1961).

27. Romanovski $V$. I. Sur quelques classes nouwels of polynomes orthogonaux. C. R. Acad. Sci. Paris, 188, 1023-1025 (1929).

28. Rosengren $H$. Multilinear Hankel forms of higher order and orthogonal polynomials. Math. Scand., 82, No. 1, 53-88 (1998).

29. Rosengren $H$. Multivariable orthogonal polynomials and coupling coefficients for discrete series representations. SIAM J. Math. Anal., 30, 233-272 (1999).

30. Tirao J. A. The matrix-valued hypergeometric equation. Proc. Natl. Acad. Sci. USA, 100, No. 14, 8138-8141 (2003).

31. Titchmarsh E. C. Eigenfunction expansions with second-order differential operators. V. 1, Clarendon Press, Oxford, 1946. [Имеется перевод: Титчмарш Э. Разложения по собственным функциям, связанные с дифференциальными уравнениями второго порядка. Т. 1. ИЛ, М., 1960.]

32. Vilenkin N. Ya., Klimyk A. U. Representations of Lie groups and special functions. Vol. 1-2. Kluwer, 1991.

33. Weyl $H$. Uber gewonliche lineare Differentialgleichungen mis singularen Stellen und ihre Eigenfunktionen (2 Note). Nachr. Konig. Gess. Wissen. Göttingen. Math.-Phys., 1910, pp. 442-467; Reprinted in Weyl H. Gessamelte Abhandlungen, Bd. 1, SpringerVerlag, 1968, pp. 222-247.

34. Zhang Gen Kai Tensor products of weighted Bergman spaces and invariant Ha-plitz operators. Math. Scand., 71, No. 1, 85-95 (1992).

ИТЭФ, группа матфизики

University of Vienna

Поступило в редакцию

email: neretin@mccme.ru 10 сентября 2003 г. 